Revista Avances en Salud 2019; 3(1): 6-7

DOI: $10.21897 / 25394622.1762$

ISSN-e 2619-4945

\title{
Definición de Salud desde la postura de Salubrista
}

\section{Definition of Health from view point of the health expert}

\author{
Álvaro A Sánchez Caraballo1 ${ }^{10}$
}

La tematización de la salud, como una cuestión filosófica, parece tener por lo menos dos justificaciones plausibles. La primera es que la salud es un tema filosófico frecuente en la época clásica. De ese asunto se han ocupado, entre otros autores, Leibniz, Diderot, Descartes, Kant y posteriormente Nietzsche. Pero, cuando se habla de salud parece ser Descartes quien se ha convertido en una referencia obligada, desde el momento en que se le atribuye la "invención de una concepción mecanicista de las funciones orgánicas" (1).

Diversos autores en diferentes momentos históricos han catalogado la salud, desde una concepción filosófica, por aludir sin ningún tipo exclusión ni priorización a Kan y Niestzsche. Sin embargo, Canguilhem sostiene que existe un concepto filosófico que aborda elementos que consideran, que el concepto de salud obedece a una "invención de una concepción mecanicista de las funciones orgánicas". De acuerdo con el autor referenciado, para establecer dicho paralelo, se debe tener en cuenta lo establecido por Descartes.

Cuando desde el área de la salud, se hacen abordajes epistemológicos para determinar la validez conceptual de un conocimiento especifico como es el de salud. Se debe entonces, recurrir a la vertiente teórica que cultive cada autor y por supuesto su concepción epistemológica y paradigmática. Desde luego, que esto le permitirá apropiarse de argumentos con criterios de objetividad, que lo pueden llevar en el mejor de los caos, a un aterrizaje hacia una o varias corrientes del pensamiento.

Es posible sostener entonces, que el concepto de Salud, como proceso vital del hombre, ha cambiado en función del tiempo y de las transformaciones del pensamiento humano. Recuérdese, que inclusive el mismo Galeno, cuestionó el concepto de salud y enfermedad a través del naturalismo hipocrático y el racionalismo. En la edad moderna, con el auge de la ciencia hay un interés natural por el cuerpo humano. En el siglo XVII la Revolución Industrial y los avances técnicos científicos de la época, consideraron aspectos biológicos y físicos, económicos, sociales y políticos relacionados con la salud. A finales del siglo XIX y comienzos del XX se concibe la salud como factor de desarrollo.

En 1946, surge la definición de salud enunciada por la Organización Mundial de la Salud (OMS): "El estado de completo bienestar físico, mental y social y no solamente la ausencia de enfermedad"(2). Un concepto utópico para la realidad dinámica de las naciones producto de los avances tecnológicos, las nuevas formas de producción, el acceso a la información y los cambios ambientales. 
Se ha hegemonizado el concepto de salud concebido como el estado de equilibrio y de adaptación a medio, en el sentido en que estas instituciones se convierten en el referente a nivel mundial para tematizarlo, y es en concordancia a esta que se establecen acciones para el direccionamiento de las políticas en salud y de las intervenciones efectivas sobre el cuerpo y la vida de los seres humanos (3). Ahora bien, frente a un hecho como la transformación del pensamiento constante se hegemonice una conceptualización de la salud.

Queda entonces un interrogante mayúsculo, ¿es posible validar o refutar el conocimiento paradigmático acerca del término salud? Considerando la conceptualización hegemonizada por la OMS y la VIII Conferencia Nacional de Salud, es posible validar el concepto de salud en el sentido en que permite la construcción de modelos, estrategias y acciones a priori para mitigar, prevenir y/o corregir las afectaciones generadas por los riesgos a los que se ve expuesta la población basándose en la realidad (el enfermo) sobre la cual trata el saber y el punto de vista desde la cual esa realidad es conocida (3). Sin embargo, esta no es una verdad absoluta e irrefutable puesto que la conceptualización de la salud es cambiante y está en constante transformación.

\section{Referencias}

1. Canguilhem G. Canguilhem y el estatuto epistemológico del concepto de salud. História, Ciências, Saúde, Manguinhos; 1997. 4(2), 287-307.

2. Amar JJ, González BM, Martínez B, Utria LU. Nuevo abordaje de la salud considerando la resiliencia. Salud Uninorte; 2013. $01 ; 29(1)$
3. García, JC. Medicina y sociedad. Las corrientes de pensamiento en el campo de la salud. Educación médica y salud; 1983. 17(4), 363-397.

${ }^{1}$ Editor Revista Avances en Salud $10.21897 / 25394622.1762$ 